\title{
People with Cerebral Palsy and Their Family's Preferences about Genomics Research
}

\author{
Yana Alexandra Wilson ${ }^{a, b}$ Sarah Mclntyre ${ }^{a, b} \quad$ Emma Waight $^{a, b}$ \\ Marelle Thornton $^{c}$ Saskia van Otterloo ${ }^{c}$ Sophie Rachel Marmont ${ }^{c}$ \\ Michael Kruer d, e Gareth Baynamf, g, h, i, j Jozef Gecz ${ }^{k, l, m} \quad$ Nadia Badawi a, b, n
}

aDiscipline of Child and Adolescent Health, Faculty of Medicine and Health, The University of Sydney, Sydney, NSW, Australia; ${ }^{b}$ Cerebral Palsy Alliance, Sydney, NSW, Australia; ${ }^{c} C P$ Quest, Cerebral Palsy Alliance, Sydney, NSW,

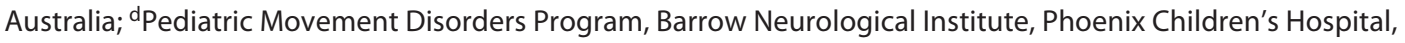
Phoenix, AZ, USA; 'Departments of Child Health, Neurology, Cellular \& Molecular Medicine and Program in

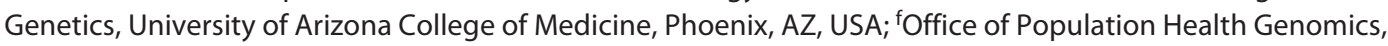
Public Health Division, Department of Health, Government of Western Australia, Perth, WA, Australia; ${ }^{9}$ School

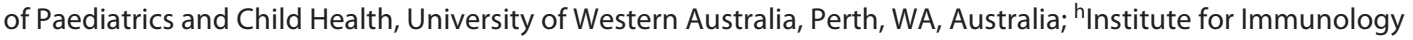
and Infectious Diseases, Murdoch University, Perth, WA, Australia; 'Telethon Kids Institute, University of Western Australia, Perth, WA, Australia; 'Spatial Sciences, Department of Science and Engineering, Curtin University, Perth, WA, Australia; ${ }^{k}$ Robinson Research Institute, The University of Adelaide, Adelaide, SA, Australia; 'Adelaide Medical School, The University of Adelaide, Adelaide, SA, Australia; 'mSouth Australian Health and Medical Sciences Institute,

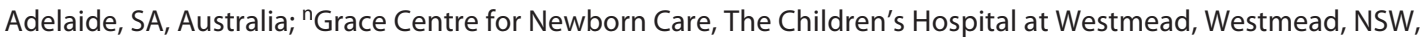
Australia

\section{Keywords}

Cerebral palsy - Genome research - Participant perspectives . Data sharing · Biobanking

\begin{abstract}
Introduction: The goal of this study was to understand individuals with cerebral palsy (CP) and their family's attitudes and preferences to genomic research, including international data sharing and biobanking. Methods: Individuals with $\mathrm{CP}$ and their family members were invited to participate in the web-based survey via email (NSW/ACT CP Register) or via posts on social media by Cerebral Palsy Alliance, CP Research Network, and CP Now. Survey responses included yes/no/
\end{abstract}

karger@karger.com www.karger.com/phg

Karger $\stackrel{\text { ' }}{5}$
(C) 2021 The Author(s)

Published by S. Karger AG, Basel

This is an Open Access article licensed under the Creative Commons Attribution-NonCommercial-4.0 International License (CC BY-NC) (http://www.karger.com/Services/OpenAccessLicense), applicable to the online version of the article only. Usage and distribution for commercial purposes requires written permission. unsure, multiple choices, and Likert scales. Fisher's exact and $X^{2}$ tests were used to assess if there were significant differences between subgroups. Results: Individuals with CP and their families ( $n=145$ ) were willing to participate in genomics research (68\%), data sharing (82\%), and biobanking efforts $(75 \%)$. This willingness to participate was associated with completion of tertiary education, previous genetic testing experience, overall higher genomic awareness, and trust in international researchers. The survey respondents also expressed ongoing communication and diverse information needs regarding the use of their samples and data. Major concerns were associated with privacy and data security. Discussion: The success of genomic research and international data sharing efforts in CP are contingent upon broad 
support and recruitment. Ongoing consultation and engagement of individuals with $\mathrm{CP}$ and their families will facilitate trust and promote increased awareness of genomics in $\mathrm{CP}$ that may in turn maximize participant uptake and recruitment.

(c) 2021 The Author(s)

Published by S. Karger AG, Basel

\section{Introduction}

Genomic technology has transformed and accelerated our understanding of human health and disease. However, genomic research requires vast quantities of data to achieve adequate sample size for analyses. To facilitate rapid discovery and translation of genomics to end care for individuals, researchers are strongly encouraged to share their participant research data more broadly. As a result, advances in genomic research depend to some extent upon large-scale international collaboration combined with biobanking and data sharing. However, ensuring that research participants and their families are adequately informed about the collection, use, and storage of their biological material and data is challenging [1].

Cerebral palsy $(\mathrm{CP})$ is a neurological condition that affects movement and posture. Historically, $\mathrm{CP}$ was thought to be caused by brain injury around the time of birth. However, studies of total populations of children with $\mathrm{CP}$ (which have occurred in the intervening years) have now firmly established that $\mathrm{CP}$ comprises many types of brain injuries/maldevelopments with etiological pathways known, uncertain, and for some still unknown [2]. Genomic research in $\mathrm{CP}$ over the last decade has demonstrated a clear and important role of genetic factors in CP etiology [reviewed in [3]). The importance of genetics for $\mathrm{CP}$ is not only in understanding precise and timely etiology, but opening future opportunities for prevention, prognosis, and personalized treatment. To aid precision medicine in CP, biobanking and data sharing platforms will form an integral part of the international CP genomics research landscape [4]. The success of these efforts will rely heavily on families' willingness to participate in genomics research and consumer/family engagement to address the complexities associated with international biobanking and data sharing. However, public consultation with individuals with $\mathrm{CP}$ and their families to elucidate attitudes, concerns, perceptions, and information needs has not yet been undertaken.

Prior studies have examined the perspectives of people with cancer, rare disease, or the general public to genomic studies; however, relatively little is known about the at- titudes of individuals with $\mathrm{CP}$ and their family members toward participation. Therefore, this survey sought to characterize the views of people with $\mathrm{CP}$ and their families to determine their: (1) willingness to participate in genomics research and what information they need to make this decision; (2) views on international data sharing and biobanking of their biological samples; (3) how they prefer to receive genomic results and research updates. The results of this study will guide the development and quality of data sharing platforms and biobanks in CP.

\section{Methods}

The study was conducted in partnership with 3 Research Partners from CP Quest, an initiative where people with CP and family members collaborate with researchers to improve the quality of research, and ensure research is meaningful for families. The 3 Research Partners are all co-authors of this study and included a young adult with CP (S.O.), an adult with CP (S.M.), and a parent of an adult with CP (M.T.).

The reporting of this survey follows the Checklist for Reporting Results of Internet E-Surveys (CHERRIES) (see online suppl. Table 1; all online supplementary material is available at www. karger.com/doi/10.1159/000518942), which aims to improve the quality of web-based survey reporting [5].

\section{Survey Development}

We reviewed the literature and conducted semi-structured interviews with our Research Partners to identify major themes to be examined in genetic studies, biobanking, and data sharing. The draft survey questions were developed, and the survey piloted, reviewed, and refined in conjunction with the Research Partners. The pilot ensured that complexity was minimized, duplications deleted, and questions reduced so that the survey was as streamlined as possible. There were a total of 71 questions, with a maximum of 8 questions per page across 15 pages. The final survey consisted of multiple-choice, Likert scale, open-ended text box questions and included adaptive questioning logic. Our items did not include a "non-response" option such as, "I'd rather not say"; however, none were compulsory. We included background information on genetics, biobanking, and data sharing for survey respondents to review. The survey was available in English only.

The introduction information for participants included the purpose of the survey, who the investigators were, how the information would be stored, and the length of time it would take to complete the survey.

The survey was open for a 3-month period, was voluntary, and no incentives were offered. The participants were individuals with $\mathrm{CP}$ and parents or carers of individuals with CP. Invitations to take part in the electronic survey were sent via email to the NSW/ACT CP Register $(n=2,300)$, CP Quest $(n=50)$, and posted on social media platforms for CP Alliance (Australia), CP Research Network (USA), CP Now Foundation (USA), and Reaching for the Stars (USA). This was a convenience sample and we are unable to identify the total number of people that the advertising of the study reached. See online suppl. Material for survey announcements. Participants were able to review and change their responses over 
Table 1. Survey participant demographics and willingness to take part in a CP genomics study

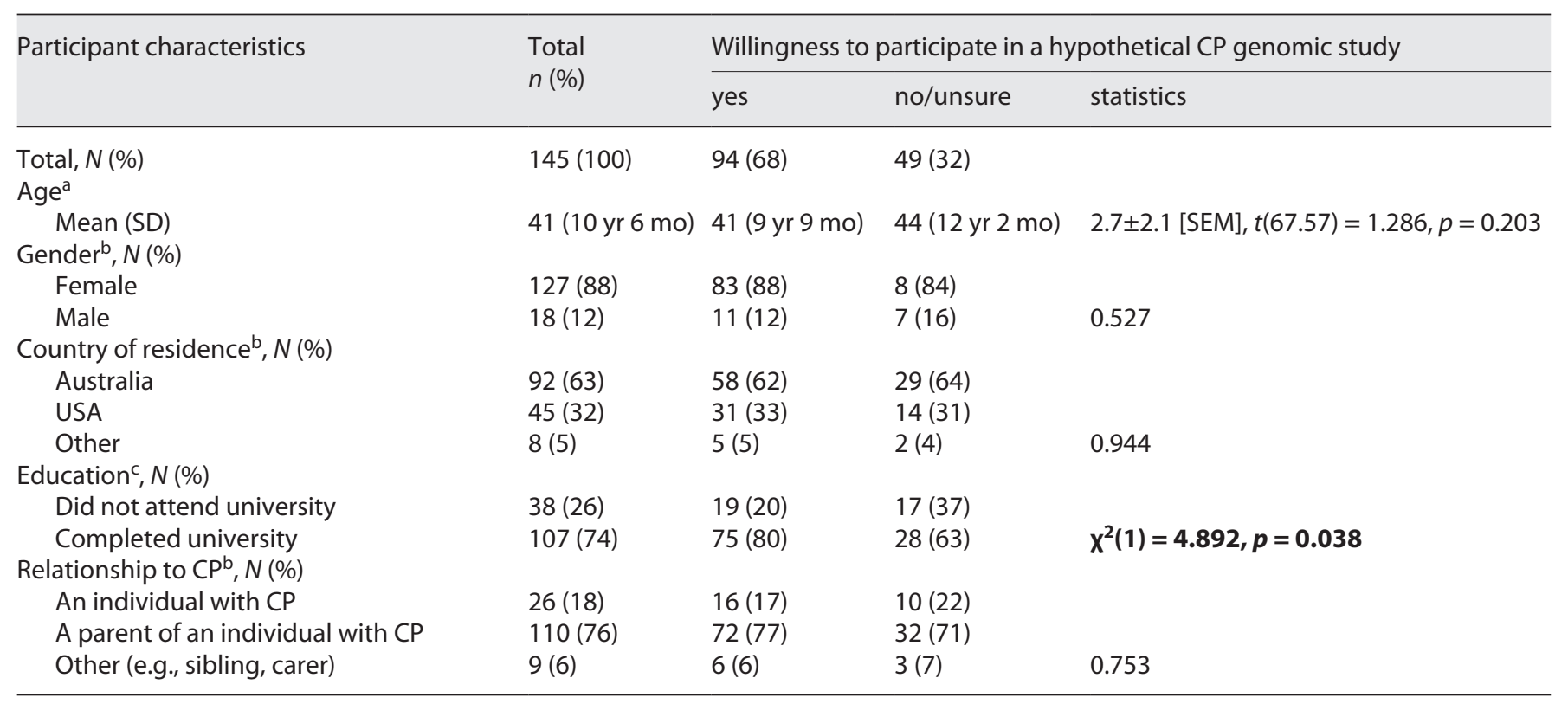

$\mathrm{CP}$, cerebral palsy; SD, standard deviation. ${ }^{\mathrm{a}}$ Independent $t$ test. ${ }^{\mathrm{b}} \mathrm{X}^{2}$ analysis. ${ }^{\mathrm{c}}$ Fisher's exact analysis.

this time period. Responses were automatically captured by REDCap. We considered the content of this survey to be low risk for multiple entries from the same person; therefore, we did not use cookies or complete IP address checks for this purpose. The project has ethics approval from the University of Sydney Human Research and Ethics Committee, a National Health and Medical Research Council accredited HREC (2020/470).

Analysis

Records were removed for analysis if demographic data were incomplete or no survey data were captured (i.e., people logged in to look at the survey but did not participate). Incomplete records, such as those in which a participant did not answer every question, were included in analysis. If $60 \%$ of respondents replied to a question, it was considered "answered" and was analyzed.

Demographics and survey responses were summarized using descriptive statistics. For some statistical analyses, we dichotomized the categorical variables into yes ("yes") versus no ("no"/"unsure"). Trust of the international research community, originally a 5-point Likert scale, was dichotomized to "do not trust" (1-2) and "do trust" (4-5). To investigate factors associated with willingness to participate, we split the respondents in those willing to participate or those unsure or unwilling to participate based on their answer to, "Would you be willing to donate a biological sample for a CP genomics study"? The genomic awareness score was developed based on the aggregated responses to 4 questions. Each question could only be answered yes, no or unsure. The questions: (1) are you familiar with the word DNA; (2) are you familiar with the word gene; (3) are you familiar with the word genome, and; (4) are you aware that your genetic testing results may have implications for your extended family. A score of zero was recorded for each question the respondent said no or unsure, and a score of 1 was recorded for each yes. The lowest possible aggre- gated score was zero and the maximum aggregated score was 4 . A score of $0-1$ was designated "no awareness," a score of 2 was assigned "limited awareness," a score of 3 was "somewhat aware," and a score of 4 was assigned "very aware."

Fisher exact test and $\chi^{2}$ tests were used to test for potential confounders between demographics. To test if there was a difference in the age of those willing to participate or not, we used an independent samples $t$ test. To test the difference between dichotomous variables and willingness to participate, we used Fisher's exact test. Likert scale responses were summarized using the package "likert" [6] in $\mathrm{R}$ (Rx64, version 3.5.0, http://www.R-project.org/). To test for differences in all other multinomial variables and willingness to participate, we used the $\chi^{2}$ test of homogeneity. All statistical tests were 2 -sided, where $p<0.05$ were considered statistically significant. Error bars are 95\% confidence intervals for all graphs. Analyses were performed using IBM SPSS Statistics 24 (IBM).

\section{Results}

\section{Participant Characteristics}

Of the 183 people that opened the survey, 145 individuals completed the demographic information and responded to the survey. Completion rate for each question ranged between 65 and 100\%. The characteristics of the participants are shown in Table 1. Our cohort was generally well-educated, predominantly female, and parents of an individual with CP. There were no significant differences between demographic measures, and they are distributed independently of each other (data not shown). A 
Table 2. Traits of survey respondents associated with willingness to participate in a CP genomics study

\begin{tabular}{|c|c|c|c|c|}
\hline \multirow[t]{2}{*}{ Participant characteristics } & \multirow{2}{*}{$\begin{array}{l}\text { Total } \\
n(\%)\end{array}$} & \multicolumn{3}{|c|}{ Willingness to participate in a hypothetical CP genomic study } \\
\hline & & $\begin{array}{l}\text { yes, } \\
n(\%)\end{array}$ & $\begin{array}{l}\text { no/unsure, } \\
n(\%)\end{array}$ & Statistics \\
\hline \multicolumn{5}{|l|}{ Previous genetic testing ${ }^{a}$} \\
\hline Yes & $40(28)$ & $33(39)$ & $7(19)$ & \multirow{3}{*}{$X^{2}(1)=4.464, p=0.037$} \\
\hline No/unsure & $80(55)$ & $51(61)$ & $29(81)$ & \\
\hline Did not respond & $25(17)$ & & & \\
\hline \multicolumn{5}{|l|}{ Genomic awareness ${ }^{b}$} \\
\hline Very aware* & $15(10)$ & $14(16)$ & $1(3)$ & \multirow{4}{*}{$x^{2}(2)=11.06, p=0.004$} \\
\hline Somewhat aware & $62(43)$ & $45(52)$ & $17(45)$ & \\
\hline Limited awareness* & $47(32)$ & $27(31)$ & $20(53)$ & \\
\hline Did not respond & $21(14)$ & & & \\
\hline \multicolumn{5}{|l|}{ Trust in researchers home $\mathrm{b}^{\mathrm{b}}$} \\
\hline Yes & $84(58)$ & $66(85)$ & $18(75)$ & \multirow{4}{*}{0.469} \\
\hline Neutral & $16(11)$ & $11(14)$ & $5(20)$ & \\
\hline No & $2(1)$ & $1(1)$ & $1(5)$ & \\
\hline Did not respond & $43(30)$ & & & \\
\hline \multicolumn{5}{|l|}{ Trust in researchers away ${ }^{b}$} \\
\hline Yes* & $61(42)$ & $52(67)$ & $9(39)$ & \multirow{4}{*}{$X^{2}(2)=13.343, p=0.001$} \\
\hline Neutral & 27 (19) & $21(27)$ & $6(26)$ & \\
\hline No* & $13(9)$ & $5(7)$ & $8(35)$ & \\
\hline Did not respond & $44(30)$ & & & \\
\hline \multicolumn{5}{|c|}{ Receiving personalized genetic results influence your decision to participate ${ }^{b}$} \\
\hline Yes* & $35(31)$ & $21(25)$ & $14(47)$ & \multirow[t]{4}{*}{$X^{2}(2)=26.394, p<0.001$} \\
\hline No* & $55(49)$ & $52(62)$ & $3(10)$ & \\
\hline Unsure & $23(20)$ & $10(12)$ & $13(43)$ & \\
\hline Did not respond & $32(22)$ & & & \\
\hline
\end{tabular}

quarter of survey participants $(n=40)$ had previous genetic testing experience and overall 53\% $(n=77)$ of the participants were "somewhat aware" or "very aware" about genomics (Table 2).

Willingness to Participate in Genomic Studies Was Associated with Higher Education Attainment, Previous Genetic Testing, Genomics Awareness, and Trust in International Researchers

When the participants were asked if they would be willing to participate in a genetic study of $\mathrm{CP}$, two-thirds of the respondents' indicated that they were willing (Table 1). Of those willing to participate, $>65 \%$ stated their reasons as "wanting to contribute to the scientific knowledge of CP." Willingness to participate in a CP genetic study was not associated with age, gender, country of residence, or the respondent's relationship to CP. However, willingness to participate was associated with tertiary education completion $\left(\chi^{2}(1)=4.892, p=0.038\right)$, previous genetic testing $\left(\chi^{2}=4.464, p=0.037\right)$, and overall genomic awareness $\left(\chi^{2}=11.060, p=0.004\right)$.

When examining whether trust in the researcher played a role in respondent's willingness to participate, we found was no difference between willingness to participate and trust in researchers from the respondents' home country $(p=0.469)$. However, we did observe a significant difference between willingness to participate and trust in the international research community $\left(\chi^{2}(2)=\right.$ 13.343, $p=0.001$ ).

Information about the Purpose of the Study and

Protecting Participant's Privacy Are the Most Important

Pieces of Information for Participants to Receive

Figure 1 shows what information respondents considered important in order to consent to participate in a CP genetics study. More than $80 \%$ of respondents noted that the most important (moderately important/very important) information they needed, when being asked to join 


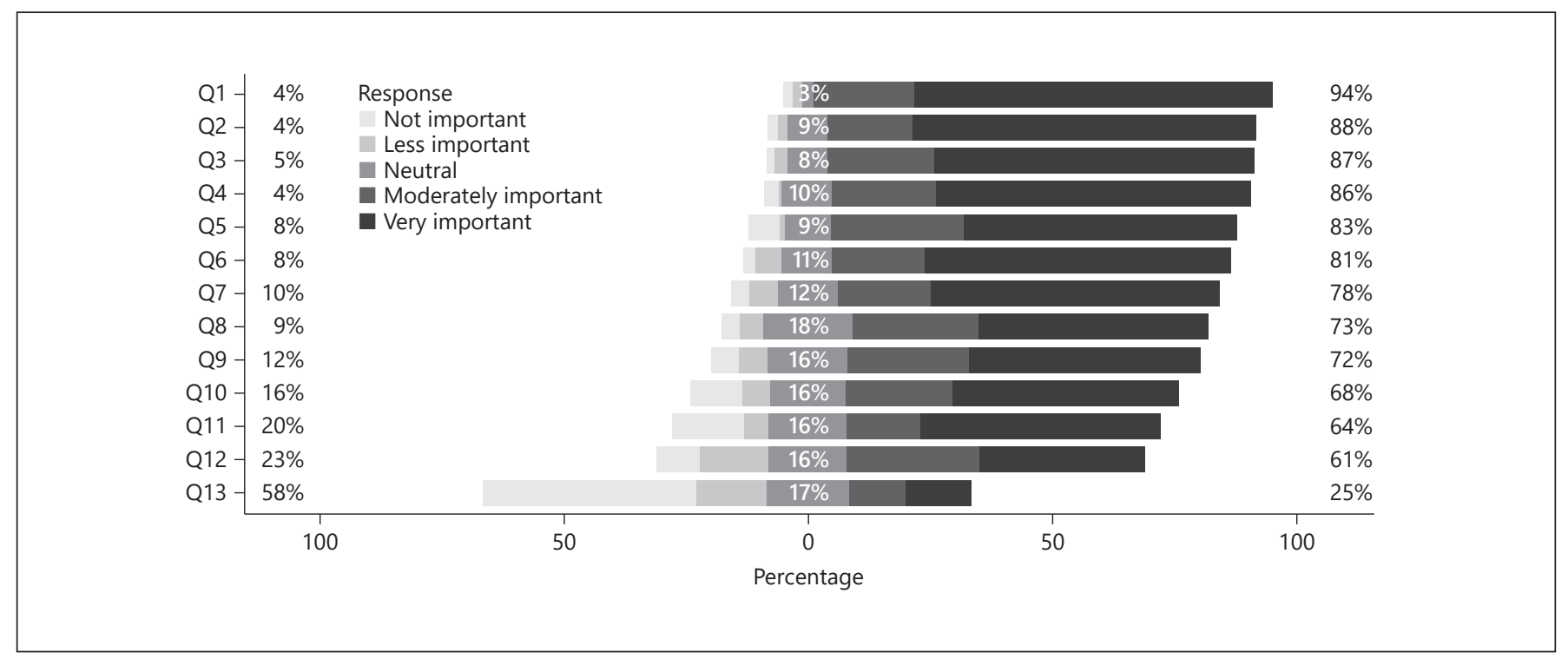

Fig. 1. Respondents rank the importance of information given in information sheets about genetic studies, biobanking, and data sharing. Q1: Purpose of the genetic study $(n=107)$, Q2: Data anonymization steps of database $(n=102), \mathbf{Q 3}$ : Confidentiality of their information in the genetic study $(n=109), \mathbf{Q 4}$ : Data breach management plan of database $(n=102), \mathbf{Q 5}$ : What research projects their de-identified data have been accessed for $(n=102), \mathbf{Q 6}$ : Risk and benefits of participating in genetic research $(n=108), \mathbf{Q 7}$ : How

a genetic study were: the purpose of the study (Q1), how their confidentiality was protected (Q2-3), what would happen in the event of a data breach (Q4), who was accessing their de-identified information (Q5), and the risks and benefits of participating (Q6).

When we considered these results based on willingness to participate, we observed differences in what information respondents' thought was important. Of those willing to participate, only Q1-4 had $>80 \%$ ranking these items as "moderately important/very important." Whereas those unsure or unwilling to participate indicated that Q1-9 and Q11 (Fig. 1) were "moderately important/very important." Information about the study purpose (Q1) was important for both groups, although those unsure or unwilling to participate were also interested in the risk and benefits of participating in genetic research (Q6) and whether they would receive their genetic results (Q11). Data security and privacy were predominant information needed by both groups (Q2-Q4), and those unsure or unwilling also indicated that information about what projects their data would be accessed for (Q5), how their data are securely stored (Q7), how researchers gain access to the database (Q8), and how they could withdraw data are securely stored, Q8: How researchers gain access to data in database $(n=102), \mathbf{Q 9}$ : Withdraw de-identified data from database $(n=102), \mathbf{Q 1 0}$ : Commercial profits $(n=125)$, Q11: Return genetic results $(n=124), \mathbf{Q 1 2}$ : Where sample processed/sequenced $(n=107)$, Q13: Acknowledged for participating $(n=108)$. Percentages from left to right represent "not important/less important," "neutral," and "moderately important/very important." from the database if they changed their mind (Q9) was also very important in their decision-making to participate.

\section{Research Participants Want Regular Communication}

Figure 2a shows that nearly $50 \%$ of survey respondents were interested in receiving updates about the research project they were recruited to every 3 months or sooner. These findings were irrespective of any participant demographics or other characteristics. The great majority of survey respondents $(95 \%, n=107)$ were interested in receiving their personalized genetic results, most of whom wanted to receive all their genetic findings, including incidental findings ( $n=98$ ) (Fig. $2 b)$. We also examined whether offering genetic results may influence a respondent to participate in a CP genomics study. Figure 2c shows that nearly $50 \%$ of respondents would not be influenced by the prospect of receiving their personalized genetic results. However, when these data were stratified by willingness to participate in a CP genomics study, we did observe that those who were unsure or unwilling to participate in a CP genomics study were more likely to be influenced by the offer of receiving their personalized re- 
sults $\chi^{2}(2)=26.394, p<0.001$ (Table 2). There was no further association between education, genome awareness, or prior genetic testing experience (data not shown).

\section{Respondents Are Supportive of Data and Sample}

Sharing, but Want to Be Kept Up-To-Date about How

Their Data or Samples Are Being Used

More than $80 \%$ of survey respondents were willing to share their de-identified data (Fig. 3a). Furthermore, when we asked respondents to specify who they would be willing to share their de-identified data with, $>60 \%$ were supportive of international data sharing (Fig. 3b). We did not find any factors associated with willingness to share data internationally, except trust in international researchers $\left(\chi^{2}(2)=33.074, p<0.001\right)$. Interestingly, $60 \%$ of respondents wanted to be informed each time a researcher wished to access their data (Fig. 3c), despite also agreeing that a third party or Ethics Committee could decide on their behalf about who could access their de-identified data (Fig. 3d). We observed the same trends regarding participants expectations to biobanking (Fig. 3e-h), although there was more uncertainty in whether or not they would allow a third party to decide on their behalf about who may access their samples.

\section{Discussion}

$\mathrm{CP}$ is a neurodevelopmental condition with complex etiologies and causal pathways. Improving our understanding of the underlying biology of $\mathrm{CP}$ using genomic

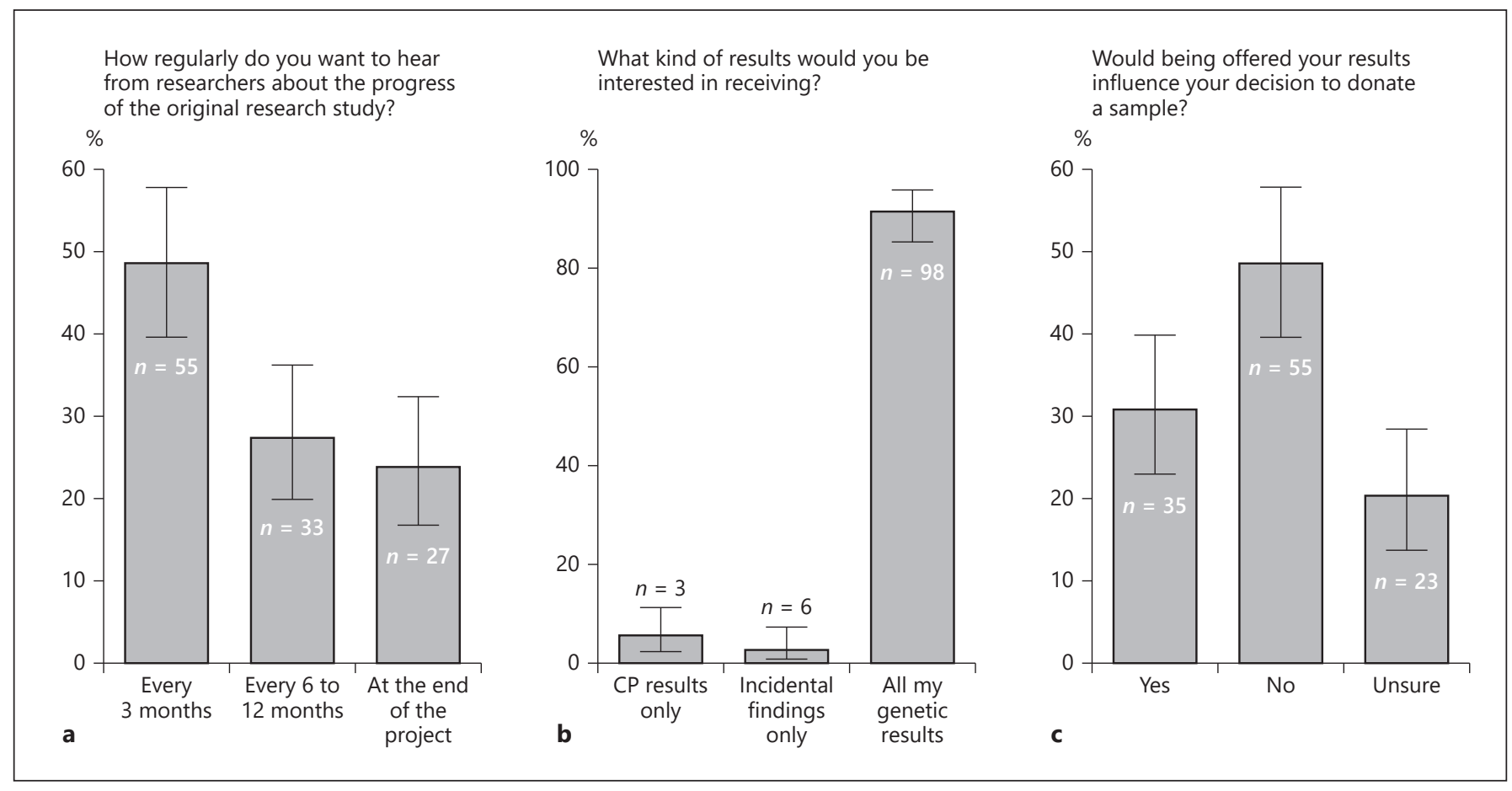

Fig. 2. Communicating with participants throughout the research project lifecycle. a Shows the communication frequency participants prefer to hear from researchers during a project $(n=115)$. b Shows what types of results participants are interested in receiving $(n=107)$. c Shows the percentage of respondents who would or would not be influenced in participating in a genetic study based on the option of receiving their genetic results $(n=$ 113). CP, cerebral palsy.

Fig. 3. Respondents attitudes toward data sharing and biobanking. $\mathbf{a}(n=104)$ and $\mathbf{e}(n=139)$ shows the percentages of participants willing or not to share their de-identified data (a) or sample $(\mathbf{e})$. b $(n=102)$ and $\mathbf{f}(n=122)$ show who the participants feel should have access to their data (b) and sample (f). $\mathbf{c}(n=99)$ and $\mathbf{g}(n=119)$ show the proportions of participants that would or would not like to be informed about researchers accessing their data (c) or samples $(\mathbf{g})$. $\mathbf{d}(n=100)$ and $\mathbf{h}(n=120)$ show the percentages of participants that would or would not allow an Ethics Committee to decide who may access their data (d) or samples (h). CP, cerebral palsy.

(For figure see next page.)
Participant Preferences about Genomics Research
Public Health Genomics 2022;25:22-31 DOI: $10.1159 / 000518942$ 


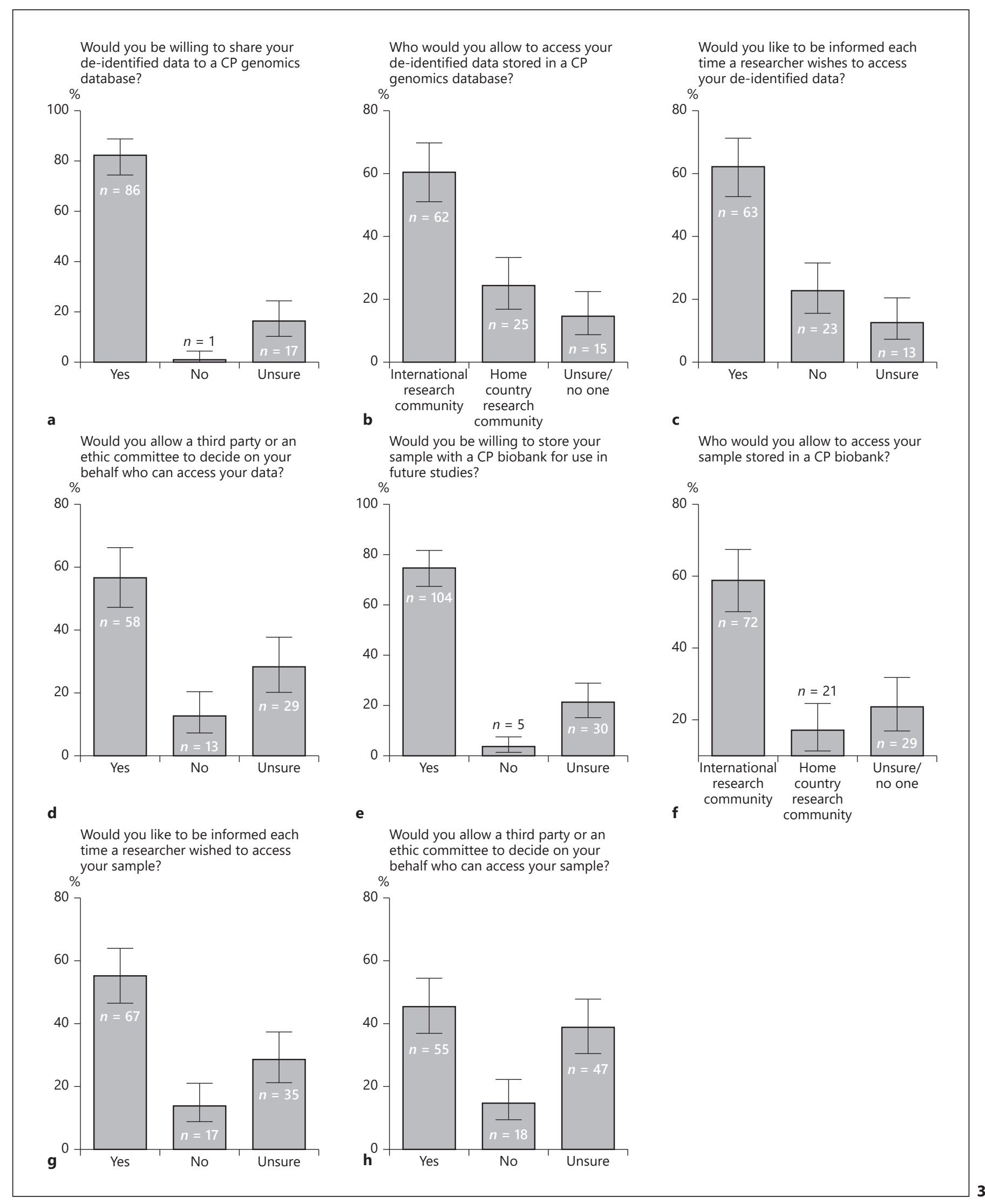


technology is contingent upon successful global collaboration and participant trust. This study examines the attitudes and perspectives of people with $\mathrm{CP}$ and their families regarding their willingness to participate in genomic research; what information is important in making these decisions; and how frequently participants want to be updated about these efforts. These results are timely given the recent establishment of the International Cerebral Palsy Genomics Consortium [7] and the rise in clinical genomics applications in neurodevelopmental disorders around the globe.

In this study, we found that more than two-thirds of respondents were willing to participate in a genomic study of CP. We observed several differences between those willing to participate compared to those unsure or unwilling to participate. Those unsure or unwilling to participate were associated with lower education attainment, no previous genetic testing experience, a lack of general awareness of genomics, and some distrust of international researchers. These findings are consistent with previous studies that show education $[8,9]$, prior genetic testing $[10,11]$, increased health literacy $[9,11]$, and trust $[9,11,12]$ are important factors in genomic research participation.

Our findings demonstrate that data security and privacy, which are tightly associated with trust $[13,14]$, are among the most important information needed for our cohort. Sanderson et al. [15] noted that in a general population cohort that the significant association between trust, privacy, and willingness to participate were abolished when participant information needs were addressed. Similarly, in a rare disease cohort, participants were highly critical of information sheets that were vague and lacking in detail [16]. Yet, in a study which offered participants increasing levels of information in the participant information sheet, only $6 \%$ accessed the most detailed information despite $>20 \%$ of participants stating they would have appreciated more detail [17]. Thus, simply providing additional information on an information sheet is unlikely to resolve the fundamental challenges when dealing with genomic literacy. Furthermore, lengthy information sheets can hinder participation, are less likely to be comprehensively read, and result in poor understanding and recall by participants $[17,18]$.

We must also acknowledge that participants do not have equal information needs and that these needs may evolve with participation and time. Many of the subsequent information needs identified as important by our respondents, and the differences we saw between those

Participant Preferences about Genomics Research willing to participate and those unsure or unwilling to participate, were associated with secondary data-use activities (i.e., who had access/what project their data contributed to). Importantly, even though many respondents were supportive of international data sharing, this willingness was contingent upon transparency and communication. Most participants in our study wanted to be informed each time a researcher requested access to their de-identified data, even if the decision to share was approved by a third party. These findings echo those from rare disease cohorts, where participants want to be informed about what their data or samples are contributing to [19-21]. While we did not investigate the reason behind this, perspectives from people with rare diseases indicate that they are interested in learning more about their conditions [22], want to ensure their data are not being misused $[16,23]$, and that recontact is crucial to building positive relationships between the researchers and participants [16]. Researchers need to consider innovative communication approaches, such as dynamic consent [24] and the use of multimedia [25] that can service the diversity of information needs throughout the research partnership. Similarly, it will be critical to develop approaches that meet the needs of genetically, culturally, and linguistically diverse populations.

Offering simple, lay language generalized results (preferably written with people with the condition), demonstrates respect toward the participant, informs participants about what was learned from the study, and enables participant feedback on findings [26]. Furthermore, communicating research outcomes with participants can build trust in genomic research, which is critical for broad recruitment and long-term sustainability of genomics activities, such as biobanks and data sharing platforms. Others have also reported that participants had greater trust in a research team due to sharing research outcomes [27] and frequent contact with participants [20]. Researchers and their funding agencies should be vigilant in upholding these standards not only because it is respectful best practice but considering distrust in the research community (following poor experiences) has been reported as a barrier to repeat participation [9] and may discourage continued participation in biobanks or data sharing initiatives.

\section{Significance}

To our knowledge, this is the first study examining the perspectives of people with CP toward genomics research. While these findings reiterate many of those from public 
and patient cohorts, denoting that people are by and large supportive of genomics efforts, we believe it is essential that people with $\mathrm{CP}$ and their family's views are represented in these discussions. While public opinion on genomics is important, there is no one size fits all model, especially in consideration for people who have lifelong conditions, like $\mathrm{CP}$. People with $\mathrm{CP}$, like those with rare diseases, have a greater vested interest in their data. These individuals, who by and large are experts through their own volition, when armed with information make better health decisions and improve their health behaviors [28]. It can also help foster collaborative relationships between the individual and health professionals [29]. As genomics becomes more frequently utilized in this diagnostic group, we encourage all researchers, clinicians, and scientists to incorporate these findings, as well as people with $\mathrm{CP}$ and their families, into their research methodology and practice.

\section{Limitations}

Our study was limited in that we captured the attitudes of potential research participants at a single time point and these opinions may change and evolve with time. Although these findings are informative, it is well known that responses to hypothetical scenarios do not always translate to real-life practices [30, 31]. Additionally, some degree of selection bias was unavoidable as we did not recruit via random sampling; therefore, potential participants who are not supportive of genetic research or could not access the internet may not have responded. However, we were pleased to capture the views of 49 people $(32 \%)$ who indicated that they were unsure or unwilling to participate in a CP genomics study. Furthermore, some degree of nonresponse bias may have been introduced to the findings as the survey questions were not compulsory.

These results have limited generalizability as our population was predominantly well-educated, female, parents from Australia. However, considering that individuals with CP and their families have previously not had an opportunity to give their opinions and attitudes regarding genetic research; this study offers an informative first step to understanding the perspective of a unique population.

\section{Conclusions}

In conclusion, our study identified several demographic and independent factors associated with a willingness to participate. The majority of our respondents were supportive of international data and biospecimen sharing; however, there are diverse information requirements from the cohort. Thus, it is crucial that we now continue to work with these groups to better understand what information is important and how we can best convey this, in order for families to not only make informed choices but to further promote trust between the participant and the researcher. The success of genomics research in CP is contingent upon broad, diverse, and culturally appropriate recruitment to ensure adequately powered sample sizes and equitable benefit. Engagement of families and people with $\mathrm{CP}$ in genomics research practice and policy will maximize participant uptake and participation, which will in turn facilitate faster breakthroughs for people with CP.

\section{Statement of Ethics}

The project has ethics approval from the University of Sydney Human Research and Ethics Committee, a National Health and Medical Research Council accredited HREC (2020/470). Written consent was not required; consent was implied following commencement of the survey.

\section{Conflict of Interest Statement}

The authors have no conflicts of interest to declare.

\section{Funding Sources}

There are no funding sources to declare.

\section{Author Contributions}

All persons who met authorship criteria are listed as authors. Y.W. and S.M. conceived the project. Y.W., S.O., M.T., S.M., and S.M. developed the survey instrument. All authors contribute to the analysis and interpretation of the findings. All authors discussed the results and the interpretation of the findings. All authors contributed, edited, and approved the final manuscript.

\section{Data Availability Statement}

The datasets generated and analysed during the study are available from the corresponding author on reasonable request.
Wilson et al. 


\section{References}

1 Rahm AK, Wrenn M, Carroll NM, Feigelson HS. Biobanking for research: a survey of patient population attitudes and understanding. J Community Genet. 2013;4:445-50.

2 Badawi N, Keogh JM. Causal pathways in cerebral palsy. J Paediatr Child Health. 2013;49: 5-8.

3 Fahey MC, MacLennan AH, Kretzschmar D, Gecz J, Kruer MC. The genetic basis of cerebral palsy. Dev Med Child Neurol. 2017;59: 462-9.

4 MacLennan AH, Kruer MC, Baynam G, Moreno-De-Luca A, Wilson YA, Zhu C, et al. Cerebral palsy and genomics: an international consortium. Dev Med Child Neurol. 2018; 60:209-10.

5 Eysenbach G. Improving the quality of web surveys: the Checklist for Reporting Results of Internet E-Surveys (CHERRIES). J Med Internet Res. 2004;6:e34.

6 Bryer J, Speerschneider K. Likert: analysis and visualization likert items. 2016. Available from: https: //CRAN.R-project.org/ package $=$ likert.

7 MacLennan AH, Kruer MC, Baynam G, Moreno-De-Luca A, Wilson YA, Zhu C, et al. Cerebral palsy and genomics: an international consortium. Dev Med Child Neurol. 2018 60:209-10.

8 Bochud M, Currat C, Chapatte L, Roth C, Mooser V. High participation rate among 25 721 patients with broad age range in a hospital-based research project involving wholegenome sequencing: the Lausanne Institutional Biobank. Swiss Med Wkly. 2017;147: w14528.

9 Henderson G, Garrett J, Bussey-Jones J, Moloney ME, Blumenthal C, Corbie-Smith G. Great expectations: views of genetic research participants regarding current and future genetic studies. Genet Med. 2008;10:193-200.

10 Middleton A, Milne R, Thorogood A, Kleiderman E, Niemiec E, Prainsack B, et al. Attitudes of publics who are unwilling to donate DNA data for research. Eur J Med Genet. 2019;62:316-23.

11 Milne R, Morley KI, Howard H, Niemiec E, Nicol D, Critchley C, et al.; Participant Values Work Stream of the Global Alliance for Genomics and Health. Trust in genomic data sharing among members of the general public in the UK, USA, Canada and Australia. Hum Genet. 2019;138:1237-46.
12 Trinidad SB, Fullerton SM, Bares JM, Jarvik GP, Larson EB, Burke W. Genomic research and wide data sharing: views of prospective participants. Genet Med. 2010;12:486-95.

13 Horn EJ, Edwards K, Terry SF. Engaging research participants and building trust. Genet Test Mol Biomarkers. 2011;15:839-40.

14 Tindana P, de Vries J, Campbell M, Littler K, Seeley J, Marshall P, et al. Community engagement strategies for genomic studies in Africa: a review of the literature. BMC Med Ethics. 2015;16:24.

15 Sanderson SC, Brothers KB, Mercaldo ND, Clayton EW, Antommaria AHM, Aufox SA, et al. Public attitudes toward consent and data sharing in biobank research: a large multi-site experimental survey in the US. Am J Hum Genet. 2017;100:414-27.

16 McCormack P, Kole A, Gainotti S, Mascalzoni D, Molster C, Lochmüller H, et al. "You should at least ask." The expectations, hopes and fears of rare disease patients on largescale data and biomaterial sharing for genomics research. Eur J Hum Genet. 2016;24:14038.

17 Antoniou EE, Draper H, Reed K, Burls A, Southwood TR, Zeegers MP. An empirical study on the preferred size of the participant information sheet in research. J Med Ethics. 2011;37:557-62.

18 Robinson JO, Slashinski MJ, Wang T, Hilsenbeck SG, McGuire AL. Participants' recall and understanding of genomic research and large-scale data sharing. J Empir Res Hum Res Ethics. 2013;8:42-52.

19 Hobbs A, Starkbaum J, Gottweis U, Wichmann HE, Gottweis H. The privacy-reciprocity connection in biobanking: comparing German with UK strategies. Public Health Genomics. 2012;15:272-84.

20 Jamal L, Sapp JC, Lewis K, Yanes T, Facio FM, Biesecker LG, et al. Research participants' attitudes towards the confidentiality of genomic sequence information. Eur J Hum Genet. 2014;22:964-8

21 Ludman EJ, Fullerton SM, Spangler L, Trinidad SB, Fujii MM, Jarvik GP, et al. Glad you asked: participants' opinions of re-consent for dbGap data submission. J Empir Res Hum Res Ethics. 2010;5:9-16.
22 Courbier S, Dimond R, Bros-Facer V. Share and protect our health data: an evidence based approach to rare disease patients' perspectives on data sharing and data protection: quantitative survey and recommendations. Orphanet J Rare Dis. 2019;14:175.

23 Long CR, Stewart MK, Cunningham TV, Warmack TS, McElfish PA. Health research participants' preferences for receiving research results. Clin Trials. 2016;13: 582-91.

24 Kaye J, Whitley EA, Lund D, Morrison M, Teare H, Melham K, et al. Dynamic consent: a patient interface for twenty-first century research networks. Eur J Hum Genet. 2015;23: 141-6.

25 Rothwell E, Johnson E, Wong B, Goldenberg A, Tarini BA, Riches N, et al. Comparison of video, app, and standard consent processes on decision-making for biospecimen research: a randomized controlled trial. J Empir Res Hum Res Ethics. 2020;15:252.

26 Beskow LM, Burke W, Fullerton SM, Sharp RR. Offering aggregate results to participants in genomic research: opportunities and challenges. Genet Med. 2012;14:490-6.

27 Purvis RS, Abraham TH, Long CR, Stewart MK, Warmack TS, McElfish PA. Qualitative study of participants' perceptions and preferences regarding research dissemination. AJOB Empir Bioeth. 2017;8:69-74.

28 Angelmar R, Berman PC. Patient empowerment and efficient health outcomes. In: Financing sustainable healthcare in Europe: new approaches for new outcomes. $2007 \mathrm{Feb}$; 1(2).p. 3.

29 Aymé S, Kole A, Groft S. Empowerment of patients: lessons from the rare diseases community. Lancet. 2008;371(9629):2048-51.

30 Holm IA, Agrawal PB, Ceyhan-Birsoy O, Christensen KD, Fayer S, Frankel LA, et al. The BabySeq project: implementing genomic sequencing in newborns. BMC Pediatr. 2018; $18: 225$.

31 Oliver JM, Slashinski MJ, Wang T, Kelly PA, Hilsenbeck SG, McGuire AL. Balancing the risks and benefits of genomic data sharing: genome research participants' perspectives. Public Health Genomics. 2012; 15:106-14
Participant Preferences about Genomics Research
Public Health Genomics 2022;25:22-31 